\title{
Stionic Effect and Seasonal Nutrient Dynamics in Some Exotic Apple Cultivars
}

\author{
J. A. Rather, F. A. Misgar, Z. A. Bhat, S. Sami Ullah, G. A. Dar and K. Javeed* \\ Division of Fruit Science Sher-e-Kashmir University of Agricultural Sciences and Technology \\ of Kashmir, Shalimar, Srinagar-19112, India \\ *Corresponding author
}

\section{A B S T R A C T}

The present studies entitled "Rootstock effects on seasonal nutrient dynamics and horticultural characteristics in some exotic apple cultivars" aimed to investigate differences of nutrient concentrations among different exotic cultivars on various rootstocks growing in the same conditions. During the investigation it was observed that leaf nutrient concentrations significantly varied with rootstocks during both the seasons.

Keywords

Apple, Rootstock, Cultivar, Seasonal variations, Nutrient concentrations

Article Info

Accepted:

04 December 2017

Available Online:

10 January 2018
The exotic cultivars like Vista Bella on $\mathrm{MM}_{106}$ recorded highest leaf $\mathrm{P}(0.21,0.20 \%)$ and highest $\mathrm{K}$ content $(1.54,1.55 \%)$ recorded in Starkrimson on both rootstocks. However, the maximum $\mathrm{Mg}$ content $(0.30,0.30 \%)$ was observed in Cooper IV on both rootstocks. Similarly lower concentration of $\mathrm{P}$ and potassium content $(0.16,0.15 \%)$ and $(1.47 \%)$ recorded in Cooper IV, and minimum $\mathrm{Mg}$ nutrient content $(0.25,0.27 \%)$ recorded in Vista Bella indicating a definite effect of rootstocks on translocation of nutrients within the plant system. The seasonal variation of leaf nutrient concentrations during growth period indicate that early maturing cultivars viz., Vista Bella and Mollies Delicious recorded increase in nutrient content upto $30^{\text {th }}$ of June, thereafter the trend decreased, while as the mid-season cultivars like Starkrimson and Cooper IV observed a similar trend upto $15^{\text {th }}$ of July and decreased thereafter. The seasonal variation of nutrients in leaves indicate stability period of various nutrients like $\mathrm{P}, \mathrm{K}$ and $\mathrm{Mg}$ in early maturing cultivars like Vista Bella and Mollies Delicious from $15^{\text {th }}$ of June to $15^{\text {th }}$ of July, while as in mid-season cultivars Starkrimson and Cooper IV the stability in nutrient concentration was recorded from $30^{\text {th }}$ June to $30^{\text {th }}$ of July indicating the appropriate leaf sampling period for these exotic cultivars as against the sampling time of existing cultivars which is from mid-July to mid-August under similar conditions.

\section{Introduction}

Mineral nutrients are greatly influenced by rootstocks, similarly different scion cultivars exhibit variable quantities of nutrients from different rootstocks. Wide fluctuation in nutrient concentration occur in tissues during growth period, however, most suitable leaf position and sampling time are those which gave rise to least variation in its mineral concentration, the nutrient accumulation curves of apple trees are good indicators of nutrient requirement in each plant development stage (Hirzel and Best, 2009). 
The knowledge of seasonal variation in leaf nutrient concentrations is necessary in order to understand the physiology of apple nutrition, and helpful in the interpretation of leaf analysis. It will used to strength the knowledge of seasonal variations in nutrient levels of leaf that would be important to accurate prescription of subsequent fertilizer additions and will play theoretical and basic roles in practical steps for production (Nachtigall et al., 2006). Nutrients are essential for the productivity and quality of different fruits; hence the determination of nutritional needs for efficient production of high quality fruit is an important aspect of nutrient management for the orchardists. Besides giving anchorage to the tree, rootstock is also responsible for the absorption of water and nutrients, storage of photosynthates and synthesis of hormones making the scion part more tolerable.

\section{Materials and Methods}

The study was conducted at Central Institute of Temperate Horticulture Srinagar, India during 2013-2014 growing seasons. Starkrimson, Cooper IV, Mollies Delicious and Vista Bella grafted on $\mathrm{M}_{9}$ dwarf and $\mathrm{MM}_{106}$ semi vigrous rootstock was used in the study. Leaf samples were taken in eight different seasons $\left(15^{\text {th }}\right.$ of May, $30^{\text {th }}$ of May, $15^{\text {th }}$ of June, $30^{\text {th }}$ of June, $15^{\text {th }}$ of July, $30^{\text {th }}$ of July, $15^{\text {th }}$ of August and $30^{\text {th }}$ of August). Before analysis, samples were washed thoroughly with fountain water, dilute acid $(0.2 \mathrm{~N} \mathrm{HCl})$ and distilled water to remove surface residues, then they were kept at $65 \pm 5^{\circ} \mathrm{C}$ until they reached to stable weight Phosphorus was estimated by vandatemolybdate colorimetric method as outlined by Jackson (1973). Potassium was determined by flame photometer and $\mathrm{Mg}$ concentrations were determined using atomicabsorption spectrophotometry Kacar et al., (1998). Statistical analysis of nutritional statues of apple plants was evaluated depending on the values given by Jones et al., (1991). Analysis of variance was performed on the data obtained from the treatments. The level of the significance (LSD at $\mathrm{P}<0.05$ ) was used in the SAS to test significance.

\section{Results and Discussion}

\section{Phosphorous}

Comparing the leaf phosphorous content of trees between two rootstocks it was observed that significantly higher leaf phosphorous content $(0.20$ and $0.19 \%)$ was recorded in trees on $\mathrm{MM}_{106}$ rootstocks compared to leaf phosphorous content $(0.18$ and $0.17 \%)$ of trees on $\mathrm{M}_{9}$ rootstocks during the study period (table 01). Among rootstocks it was observed that semi vigrous rootstock, $\mathbf{M M}_{106}$ had significantly higher phosphorous content compared to dwarf rootstocks $\mathbf{M}_{9}$. The lower leaf nutrient concentrations in trees of $\mathrm{M}_{9}$ rootstock could be due to less vigor of these trees Kucukyumuk and Erdal (2009), the observations are also in accordance with the findings of Wustcher and Shull (1976).

Among different cultivars maximum leaf phosphorous content $(0.20$ and $0.19 \%)$ was recorded in Vista Bella whereas minimum leaf phosphorous content $(0.16 \%)$ was recorded in Cooper IV during both the years respectively. Trees of 'Delicious' and 'Golden Delicious' growing on $\mathrm{MM}_{106}$ rootstocks had higher phosphorous levels than similar trees on $\mathrm{MM}_{111}$ rootstock (Schneider et al., 1978). However, the rootstock and variety effects on nutrient concentration of apple trees can be explained with the genetic effect leading to different nutrient uptake capacity Jimenez et al., (2007). Leaf phosphorous content recorded a significant difference on both the rootstocks at different sampling dates between spring and autumn season flushes, the highest leaf phosphorous content (0.24 and $0.22 \%)$ 
was observed on $30^{\text {th }}$ of June and $15^{\text {th }}$ of July whereas, minimum leaf phosphorous content $(0.13$ and $0.12 \%)$ was recorded on $15^{\text {th }}$ of May during both the years. The higher concentration of phosphorous during the initial growth of the apple plant may be due to current absorption and also as a result of remobilization of the phosphorous and in persimmon phosphorous content of leaves decreased with the advancement of growing season Rehalia and Sandhu (2005).

\section{Seasonal variation}

Least variation period in early maturing cultivars Vista Bella and Mollies Delicious was observed on $15^{\text {th }}$ of June to $15^{\text {th }}$ of July because there is a relative nutrient concentration stability from $8^{\text {th }}$ to $12^{\text {th }}$ week after full bloom in early maturing cultivars where as in mid maturing cultivars Starkrimson and Cooper IV observed on $30^{\text {th }}$ of June to $30^{\text {th }}$ of July, $10^{\text {th }}$ to $14^{\text {th }}$ week after full bloom and might be due to least requirement of phosphorous by the growing fruit during this period and minimum changes in fully developed leaves (Fig. 1). The variation in nutrient stability period in the cultivars of apple may be due to genotypic effect.

The variation in the stability period in different crops and varieties may be due to the requirement of phosphorous during different periods of crop growth Kamboj et al., (1987).

\section{Potassium}

As for as rootstocks are concerned significantly higher leaf potassium content (1.52 and $1.54 \%$ ) was recorded in trees on $\mathrm{MM}_{106}$ rootstock compared to leaf potassium content (1.46 and $1.50 \%$ ) on $\mathrm{M}_{9}$ rootstock. Among rootstocks it was clearly seen that semi vigrous rootstock, MM $_{106}$ had significantly higher mineral nutrients, compared to dwarf rootstocks $\mathrm{M}_{9}$. Dwarf rootstocks such as $\mathbf{M}_{9}$ and $\mathbf{M}_{26}$ have smaller root systems, so it can be the major reason for having lower nutrients compared to others (Erdal et al., 2008). Among cultivars significantly maximum leaf potassium content (1.52 and $1.54 \%$ ) was recorded in Starkrimson and minimum $(1.47 \%)$ and $(1.50 \%)$ was recorded in Vista Bella and Mollies Delicious (table 02). Leaf potassium content showed a significant difference on various sampling dates, maximum leaf potassium content (1.68 and $1.71 \%$ ) was recorded on $15^{\text {th }}$ of July and minimum leaf potassium content (1.24 and $1.30 \%$ ) was recorded on $15^{\text {th }}$ of May during both the years respectively. Leaf potassium content of different cultivars on $\mathrm{M}_{9}$ and $\mathrm{MM}_{106}$ rootstocks depicted an increasing trend from $15^{\text {th }}$ of May to $15^{\text {th }}$ of July, thereafter, it decreased gradually upto $30^{\text {th }}$ of August indicating a significant difference between spring and autumn season flushes. In July and August sampling seasons potassium concentrations had the highest level compared to the other sampling dates during the season Roca-Perez et al., (2006). Potassium content of leaves sampled from different cultivars of apple increased steadily during initial period of growth and reached to maximum on $15^{\text {th }}$ of July and there after declined towards the end of sampling season Kucukyumuk et al., (2012). The decline in potassium content is associated with growth dilution effects and also due to utilization of potassium by growing points (Kotur and Singh, 1993).

\section{Seasonal variation}

The nutrient stability period with regard to early season maturing cultivars was recorded from $30^{\text {th }}$ of May to $30^{\text {th }}$ of June in Vista Bella and $15^{\text {th }}$ of June to $15^{\text {th }}$ July in Mollies Delicious whereas mid maturing cultivars Starkrimson and Cooper IV showed least variation period from $30^{\text {th }}$ June to $30^{\text {th }}$ July (Fig. 2). 
Table.1 Rootstock and cultivar effect on seasonal variation of leaf Phosphorous (\%) content of exotic apple cultivars

\begin{tabular}{|c|c|c|c|c|c|c|c|c|c|c|c|c|c|c|c|c|c|c|c|c|}
\hline \multirow{3}{*}{\begin{tabular}{|c|} 
Rootstock \\
\\
$\begin{array}{c}\text { variety } \\
\text { period }\end{array}$ \\
\end{tabular}} & \multicolumn{10}{|c|}{ Year 2013} & \multicolumn{10}{|c|}{ Year 2014} \\
\hline & \multicolumn{5}{|c|}{$\mathrm{M}_{9}$} & \multicolumn{5}{|c|}{$\mathrm{MM}_{106}$} & \multicolumn{5}{|c|}{$\mathrm{M}_{9}$} & \multicolumn{5}{|c|}{$\mathrm{MM}_{106}$} \\
\hline & SK & C.IV & MD & VB & $\begin{array}{l}\text { Sub } \\
\text { mean }\end{array}$ & SK & C.IV & MD & VB & $\begin{array}{c}\text { Sub } \\
\text { mean }\end{array}$ & SK & C.IV & MD & VB & $\begin{array}{l}\text { Sub } \\
\text { mean }\end{array}$ & SK & C.IV & $\mathrm{MD}$ & VB & $\begin{array}{c}\text { Sub } \\
\text { mean }\end{array}$ \\
\hline $15^{\text {th }}$ May & 0.13 & 0.11 & 0.12 & 0.14 & 0.13 & 0.14 & 0.11 & 0.15 & 0.16 & 0.14 & 0.12 & 0.10 & 0.13 & 0.14 & 0.12 & 0.14 & 0.10 & 0.15 & 0.16 & 0.14 \\
\hline $30^{\mathrm{TH}}$ May & 0.15 & 0.13 & 0.15 & 0.24 & 0.16 & 0.16 & 0.14 & 0.17 & 0.16 & 0.15 & 0.14 & 0.11 & 0.14 & 0.15 & 0.13 & 0.15 & 0.11 & 0.16 & 0.24 & 0.16 \\
\hline $15^{\text {th }}$ June & 0.17 & 0.15 & 0.25 & 0.27 & 0.21 & 0.20 & 0.16 & 0.24 & 0.25 & 0.21 & 0.15 & 0.12 & 0.22 & 0.21 & 0.17 & 0.18 & 0.13 & 0.23 & 0.26 & 0.20 \\
\hline $30^{\text {th }}$ June & 0.21 & 0.19 & 0.29 & 0.24 & 0.23 & 0.23 & 0.20 & 0.30 & 0.29 & 0.25 & 0.21 & 0.17 & 0.24 & 0.23 & 0.21 & 0.23 & 0.19 & 0.25 & 0.23 & 0.22 \\
\hline $15^{\text {th }}$ July & 0.23 & 0.21 & 0.23 & 0.22 & 0.22 & 0.25 & 0.22 & 0.26 & 0.25 & 0.24 & 0.23 & 0.20 & 0.23 & 0.22 & 0.22 & 0.24 & 0.21 & 0.24 & 0.21 & 0.22 \\
\hline $30^{\text {th }}$ July & 0.21 & 0.20 & 0.20 & 0.19 & 0.20 & 0.23 & 0.20 & 0.22 & 0.22 & 0.21 & 0.21 & 0.20 & 0.21 & 0.20 & 0.20 & 0.23 & 0.20 & 0.22 & 0.19 & 0.21 \\
\hline $15^{\text {th }}$ Aug. & 0.20 & 0.17 & 0.18 & 0.16 & 0.17 & 0.21 & 0.18 & 0.21 & 0.21 & 0.20 & 0.19 & 0.17 & 0.16 & 0.15 & 0.16 & 0.20 & 0.18 & 0.18 & 0.17 & 0.18 \\
\hline $30^{\text {th }}$ Aug. & 0.15 & 0.13 & 0.16 & 0.15 & 0.15 & 0.16 & 0.16 & 0.17 & 0.16 & 0.15 & 0.15 & 0.13 & 0.15 & 0.15 & 0.14 & 0.18 & 0.16 & 0.18 & 0.14 & 0.16 \\
\hline Mean (V) & 0.18 & 0.16 & 0.18 & 0.20 & & 0.19 & 0.17 & 0.21 & 0.21 & & 0.18 & 0.15 & 0.18 & 0.18 & & 0.19 & 0.16 & 0.20 & 0.20 & \\
\hline Mean (S) & \multicolumn{5}{|c|}{0.18} & \multicolumn{5}{|l|}{0.20} & \multicolumn{4}{|c|}{0.17} & & \multicolumn{4}{|c|}{0.19} & \\
\hline \multicolumn{11}{|c|}{ Factor mean varieties $\mathrm{SK}=0.18 \mathrm{C} . \mathrm{IV}=0.16 \mathrm{MD}=0.20 \mathrm{VB}=0.20$} & \multicolumn{10}{|c|}{ Factor mean $\mathrm{SK}=0.18 \mathrm{C} \mathrm{IV}=0.16 \mathrm{MD}=0.19 \mathrm{VB}=0.19$} \\
\hline \multicolumn{11}{|c|}{$\begin{array}{l}\text { CD }(p \leq 0.05) \text { Timing }(T)=0.005 \text { Rootstock }(S)=0.002 \text { Varieties }(V)=0.003 \\
\text { TXS 0.006 VXT }=0.009 \text { VXS } 0.005 \text { VXSXT }=0.01 \\
\text { Legend SK= Starkrimson C.IV= Cooper IV MD= Mollies Delicious VB= } \\
\text { Vista Bella }\end{array}$} & \multicolumn{10}{|c|}{$\begin{array}{l}\text { CD }(p \leq 0.05) \operatorname{Timing}(T)=0.00 \text { Rootstock }(S)=0.002 \text { Varieties }(V)= \\
0.003 \text { TXS 0.007 VXT }=0.010 \text { VXS NS VXSXT }=\text { NS }\end{array}$} \\
\hline
\end{tabular}


Table.2 Rootstock and cultivar effect on seasonal variation of leaf Potassium (\%) content of exotic apple cultivars

\begin{tabular}{|c|c|c|c|c|c|c|c|c|c|c|c|c|c|c|c|c|c|c|c|c|}
\hline Rootstock & & & & & Year & 2013 & & & & & & & & & Year & 2014 & & & & \\
\hline & & & $\mathrm{M}_{9}$ & & & & & $\mathrm{MM}_{1}$ & & & & & $\mathrm{M}_{9}$ & & & & & $\mathrm{MM}_{10}$ & & \\
\hline $\begin{array}{l}\text { variety } \\
\text { period }\end{array}$ & SK & C.IV & MD & VB & $\begin{array}{l}\text { Sub } \\
\text { mean }\end{array}$ & SK & C.IV & MD & VB & $\begin{array}{c}\text { Sub } \\
\text { mean }\end{array}$ & SK & C.IV & MD & VB & $\begin{array}{l}\text { Sub } \\
\text { mean }\end{array}$ & SK & C.IV & MD & VB & $\begin{array}{c}\text { Sub } \\
\text { mean }\end{array}$ \\
\hline $15^{\text {th }}$ May & 1.28 & 1.15 & 1.27 & 1.25 & 1.24 & 1.31 & 1.32 & 1.37 & 1.38 & 1.34 & 1.30 & 1.28 & 1.27 & 1.35 & 1.30 & 1.37 & 1.43 & 1.45 & 1.44 & 1.42 \\
\hline $30^{\mathrm{TH}}$ May & 1.36 & 1.27 & 1.52 & 1.52 & 1.41 & 1.41 & 1.40 & 1.43 & 1.41 & 1.41 & 1.47 & 1.36 & 1.39 & 1.59 & 1.45 & 1.47 & 1.51 & 1.51 & 1.55 & 1.51 \\
\hline $15^{\text {th }}$ June & 1.49 & 1.42 & 1.54 & 1.62 & 1.51 & 1.52 & 1.50 & 1.59 & 1.70 & 1.57 & 1.53 & 1.49 & 1.58 & 1.77 & 1.59 & 1.53 & 1.54 & 1.56 & 1.80 & 1.60 \\
\hline $30^{\text {th }}$ June & 1.52 & 1.45 & 1.75 & 1.59 & 1.57 & 1.57 & 1.54 & 1.74 & 1.73 & 1.64 & 1.57 & 1.49 & 1.75 & 1.58 & 1.59 & 1.62 & 1.63 & 1.80 & 1.65 & 1.67 \\
\hline $15^{\text {th }}$ July & 1.73 & 1.71 & 1.60 & 1.52 & 1.64 & 1.74 & 1.72 & 1.69 & 1.59 & 1.68 & 1.75 & 1.70 & 1.55 & 1.53 & 1.63 & 1.81 & 1.77 & 1.65 & 1.62 & 1.71 \\
\hline $30^{\text {th }}$ July & 1.62 & 1.52 & 1.55 & 1.49 & 1.54 & 1.62 & 1.62 & 1.56 & 1.59 & 1.59 & 1.66 & 1.61 & 1.55 & 1.48 & 1.57 & 1.61 & 1.63 & 1.55 & 1.53 & 1.58 \\
\hline $15^{\text {th }}$ Aug. & 1.55 & 1.47 & 1.32 & 1.35 & 1.42 & 1.56 & 1.51 & 1.54 & 1.57 & 1.54 & 1.61 & 1.50 & 1.51 & 1.41 & 1.50 & 1.51 & 1.47 & 1.52 & 1.51 & 1.50 \\
\hline $30^{\text {th }}$ Aug. & 1.52 & 1.50 & 1.20 & 1.15 & 1.34 & 1.57 & 1.49 & 1.16 & 1.17 & 1.35 & 1.51 & 1.48 & 1.27 & 1.20 & 1.36 & 1.46 & 1.48 & 1.20 & 1.21 & 1.34 \\
\hline Mean (V) & 1.51 & 1.44 & 1.47 & 1.44 & & 1.54 & 1.51 & 1.51 & 1.50 & & 1.55 & 1.47 & 1.48 & 1.49 & & 1.54 & 1.56 & 1.53 & 1.54 & \\
\hline Mean (S) & & & 1.46 & & & & & & & & & & & & & & & & & \\
\hline Factor me & n var & eties $\mathbf{S}$ & $=1.5$ & 2 C.IV & $=1.47 \mathrm{I}$ & $\mathrm{AD}=1$ & $49 \mathrm{~V}$ & $=1.47$ & & & Facto & mean & $\mathrm{SK}=$ & $54 \mathrm{C}$ & $V=1.5$ & $\mathrm{MD}=$ & 1.50 & $B=1.5$ & & \\
\hline $\begin{array}{l}\text { CD }(p \leq 0.0 \\
\text { TXS } 0.025 \\
\text { Legend SK } \\
\text { Vista Bella }\end{array}$ & $\begin{array}{l}\mathrm{Tim} \\
\mathrm{VXT}=\end{array}$ & $\begin{array}{l}\operatorname{lng}(\mathrm{T}) \\
0.036\end{array}$ & $\begin{array}{l}=0.018 \\
\text { XSS 0. } \\
\text { n C.IV }\end{array}$ & $\begin{array}{l}\text { Root } \\
018 \mathrm{~V} \\
=\mathrm{Cod}\end{array}$ & $\begin{array}{l}\operatorname{tock}(\mathrm{S}) \\
\text { KSXT= } \\
\text { per IV }\end{array}$ & $\begin{array}{l}=0.00 \\
0.051 \\
\mathrm{MD}=1\end{array}$ & & & & & $\begin{array}{l}\text { CD ( } \\
0.014\end{array}$ & $\begin{array}{l}\leq 0.05 \\
\text { TXS }\end{array}$ & $\begin{array}{l}\text { Timin } \\
.027 \mathrm{~V}\end{array}$ & $\begin{array}{l}\mathrm{g}(\mathrm{T})= \\
\mathrm{XT}=0\end{array}$ & $\begin{array}{l}0.019 \mathrm{~F} \\
038 \mathrm{~V}\end{array}$ & $\begin{array}{l}\text { ootsto } \\
\text { S } 0.01\end{array}$ & $\begin{array}{l}\mathrm{ck}(\mathrm{S})= \\
9 \mathrm{VX}\end{array}$ & $\begin{array}{l}0.010 \\
X T=0\end{array}$ & $\begin{array}{l}\text { Jarieti } \\
054\end{array}$ & $s(V)=$ \\
\hline
\end{tabular}


Table.3 Rootstock and cultivar effect on seasonal variation of leaf magnesium (\%) content of exotic apple cultivars

\begin{tabular}{|c|c|c|c|c|c|c|c|c|c|c|c|c|c|c|c|c|c|c|c|c|}
\hline \multirow[t]{2}{*}{ Rootstock } & \multicolumn{10}{|c|}{ Year 2013} & \multicolumn{10}{|c|}{ Year 2014} \\
\hline & \multicolumn{5}{|c|}{$\mathrm{M}_{9}$} & \multicolumn{5}{|c|}{$\mathrm{MM}_{106}$} & \multicolumn{5}{|c|}{$\mathrm{M}_{9}$} & \multicolumn{5}{|c|}{$\mathrm{MM}_{106}$} \\
\hline $\begin{array}{l}\text { variety } \\
\text { period }\end{array}$ & SK & C.IV & MD & VB & $\begin{array}{c}\text { Sub } \\
\text { mean }\end{array}$ & SK & C.IV & MD & VB & $\begin{array}{c}\text { Sub } \\
\text { mean }\end{array}$ & SK & C.IV & MD & VB & $\begin{array}{c}\text { Sub } \\
\text { mean }\end{array}$ & SK & C.IV & MD & VB & $\begin{array}{c}\text { Sub } \\
\text { mean }\end{array}$ \\
\hline $15^{\text {th }}$ May & 0.21 & 0.22 & 0.21 & 0.20 & 0.21 & 0.26 & 0.24 & 0.27 & 0.26 & 0.26 & 0.21 & 0.24 & 0.22 & 0.20 & 0.22 & 0.23 & 0.25 & 0.29 & 0.22 & 0.24 \\
\hline $30^{\mathrm{TH}}$ May & 0.22 & 0.27 & 0.22 & 0.22 & 0.22 & 0.26 & 0.30 & 0.27 & 0.27 & 0.27 & 0.22 & 0.26 & 0.23 & 0.22 & 0.23 & 0.26 & 0.25 & 0.29 & 0.24 & 0.26 \\
\hline $15^{\text {th }}$ June & 0.22 & 0.24 & 0.25 & 0.23 & 0.23 & 0.28 & 0.33 & 0.29 & 0.28 & 0.29 & 0.24 & 0.30 & 0.25 & 0.24 & 0.29 & 0.27 & 0.27 & 0.29 & 0.25 & 0.27 \\
\hline $30^{\text {th }}$ June & 0.24 & 0.26 & 0.26 & 0.32 & 0.27 & 0.29 & 0.31 & 0.29 & 0.30 & 0.29 & 0.26 & 0.33 & 0.27 & 0.33 & 0.29 & 0.29 & 0.30 & 0.31 & 0.33 & 0.30 \\
\hline $15^{\text {th }}$ July & 0.28 & 0.33 & 0.35 & 0.30 & 0.31 & 0.29 & 0.32 & 0.32 & 0.29 & 0.30 & 0.31 & 0.37 & 0.37 & 0.30 & 0.33 & 0.32 & 0.35 & 0.35 & 0.31 & 0.32 \\
\hline $30^{\text {th }}$ July & 0.32 & 0.36 & 0.33 & 0.27 & 0.32 & 0.31 & 0.33 & 0.30 & 0.29 & 0.30 & 0.34 & 0.39 & 0.34 & 0.30 & 0.34 & 0.35 & 0.36 & 0.35 & 0.30 & 0.34 \\
\hline $15^{\text {th }}$ Aug. & 0.29 & 0.35 & 0.30 & 0.26 & 0.30 & 0.29 & 0.30 & 0.29 & 0.28 & 0.29 & 0.32 & 0.35 & 0.31 & 0.27 & 0.31 & 0.31 & 0.34 & 0.32 & 0.27 & 0.31 \\
\hline $30^{\text {th }}$ Aug. & 0.27 & 0.30 & 0.27 & 0.24 & 0.27 & 0.25 & 0.26 & 0.24 & 0.23 & 0.25 & 0.29 & 0.30 & 0.28 & 0.26 & 0.28 & 0.26 & 0.30 & 0.28 & 0.27 & 0.28 \\
\hline Mean (V) & 0.26 & 0.28 & 0.27 & 0.25 & & 0.28 & 0.30 & 0.29 & 0.27 & & 0.27 & 0.32 & 0.28 & 0.27 & & 0.28 & 0.30 & 0.31 & 0.27 & \\
\hline $\begin{array}{l}\text { Factor mes } \\
\text { CD (p } \leq 0.0 \\
0.01 \text { VXT }= \\
\text { Legend SK } \\
\text { Vista Bella }\end{array}$ & $\begin{array}{l}\text { T var } \\
\text { Tim } \\
\text { NS V } \\
=\text { Sta }\end{array}$ & $\begin{array}{l}\text { ties S } \\
\text { ng (T) } \\
\text { S N NS } \\
\text { srimsi }\end{array}$ & $\begin{array}{l}=0.2 \\
0.13 \\
X S X\end{array}$ & $\begin{array}{l}\text { C.IV } \\
\text { Rootst } \\
T=\text { NS } \\
=\mathrm{CoO}\end{array}$ & $\begin{array}{l}=0.291 \\
\operatorname{ck}(S)=\end{array}$ & $\begin{array}{l}\mathrm{AD}=1 \\
0.00\end{array}$ & $\begin{array}{l}28 \mathrm{VB} \\
\text { Varie }\end{array}$ & $\begin{array}{l}=0.27 \\
\operatorname{ies}(V)\end{array}$ & 0.00 & TXS & $\begin{array}{l}\text { Facto } \\
\text { CD (1 } \\
\text { NS T }\end{array}$ & $\begin{array}{l}r \text { mear } \\
\leq 0.05 \\
X S 0.0\end{array}$ & $\begin{array}{l}\mathrm{SK}=0 \\
\mathrm{Timin} \\
0 \mathrm{VX}\end{array}$ & $\begin{array}{l}28 \mathrm{C} \mathrm{I} \\
(\mathrm{T})= \\
=\mathrm{NS}\end{array}$ & $\begin{array}{l}\mathrm{V}=0.3 \\
0.015 \mathrm{~F} \\
\mathrm{VXS} 0\end{array}$ & $\begin{array}{l}\mathrm{MD}= \\
\text { ootsto } \\
015 \mathrm{~V})\end{array}$ & $\begin{array}{l}0.30 \mathrm{~V} \\
\mathrm{k}(\mathrm{S})= \\
\mathrm{SXT}=\end{array}$ & $\begin{array}{l}\mathrm{B}=0.2 \\
\mathrm{NS} \mathrm{Va} \\
\mathrm{NS}\end{array}$ & 7 & $V)=$ \\
\hline
\end{tabular}


Table.4 Rootstock effect on fruit yield, yield efficiency in various exotic cultivars of apple and relationship between leaf nutrients with fruit quality parameters

\begin{tabular}{|c|c|c|c|c|c|c|c|c|c|c|c|c|}
\hline \multirow{4}{*}{$\begin{array}{l}\text { Treatme } \\
\text { nt }\end{array}$} & \multicolumn{6}{|c|}{ Yield (kg/tree) } & \multicolumn{6}{|c|}{ Yield efficiency $\left(\mathrm{kg} / \mathrm{cm}^{-2}\right)$} \\
\hline & \multicolumn{3}{|c|}{ Year 2013} & \multicolumn{3}{|c|}{ Year 2014} & \multicolumn{3}{|c|}{ Year 2013} & \multicolumn{3}{|c|}{2014} \\
\hline & \multicolumn{2}{|c|}{ Rootstock } & \multirow[t]{2}{*}{ Mean } & \multicolumn{2}{|c|}{ Rootstock } & \multirow[t]{2}{*}{ Mean } & \multicolumn{2}{|c|}{ Rootstock } & \multirow[t]{2}{*}{ Mean } & \multicolumn{2}{|c|}{ Rootstock } & \multirow[t]{2}{*}{ Mean } \\
\hline & $\mathrm{M}_{9}$ & $\mathrm{MM}_{106}$ & & $\mathrm{M}_{9}$ & $\mathrm{MM}_{106}$ & & $\mathrm{M}_{9}$ & $\mathrm{MM}_{106}$ & & $\mathrm{M}_{9}$ & $\mathrm{MM}_{106}$ & \\
\hline SK & 13.83 & 19.43 & 16.6 & 20.93 & 23.90 & 22.41 & 0.17 & 0.24 & 0.20 & 0.24 & 0.27 & 0.25 \\
\hline$\overline{\text { C.IV }}$ & 8.30 & 6.26 & 7.2 & 15.00 & 10.63 & 12.81 & 0.18 & 0.08 & 0.13 & 0.31 & 0.14 & 0.22 \\
\hline$\overline{\mathrm{MD}}$ & 18.71 & 15.56 & 17.1 & 15.96 & 21.76 & 18.86 & 0.29 & 0.23 & 0.26 & 0.21 & 0.29 & 0.25 \\
\hline $\mathbf{V B}$ & 14.33 & 24.93 & 19.6 & 22.70 & 24.46 & 23.58 & 0.33 & 0.32 & 0.32 & 0.27 & 0.37 & 0.32 \\
\hline Mean & 13.79 & 16.55 & & 18.65 & 20.19 & & 0.24 & 0.21 & & 0.25 & 0.26 & \\
\hline \multicolumn{7}{|c|}{$\begin{array}{l}\text { CD }(p \leq 0.05) \\
2013=R \text { 1.47 V } 2.08 \text { RXV } 2.94 \\
2014=\text { R NS V 2.55 RXV 3.60 } \\
\text { Legend= SK= Starkrimson C.IV= Cooper IV MD= Mollies Delicious VB= } \\
\text { VistaBella } \\
\text { R= Rootstock, V= variety, }\end{array}$} & \multicolumn{6}{|c|}{$\begin{array}{l}2013=\mathrm{R} 0.00 \mathrm{~V} 0.00 \mathrm{RXV} 0.00 \\
2014=\mathrm{R} 0.006 \mathrm{~V} 0.008 \mathrm{RXV} 0.012\end{array}$} \\
\hline
\end{tabular}




\section{Rootstock and cultivar effect on seasonal variation of leaf nutrient content of exotic apple cultivars}

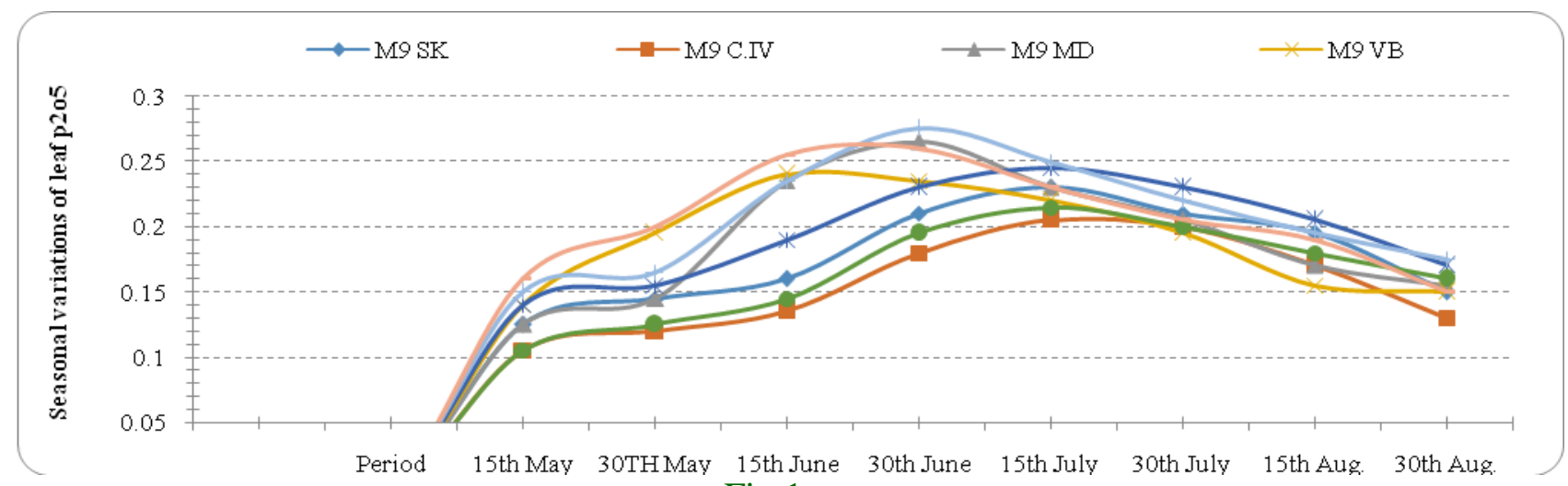

Fig.1

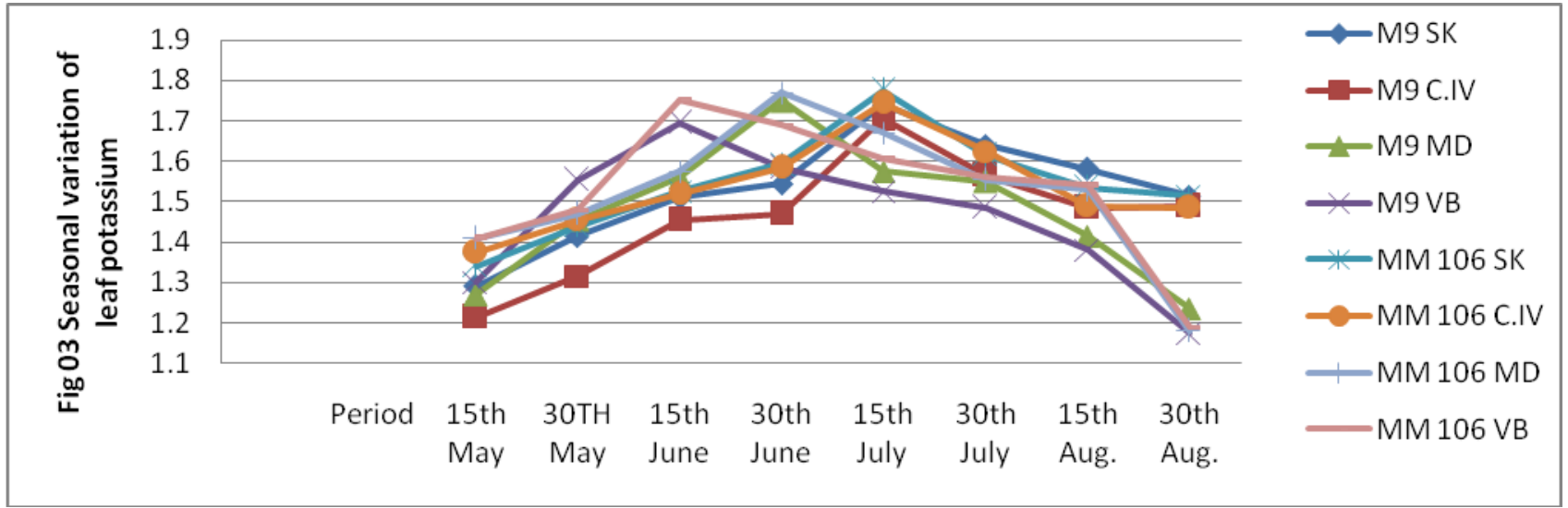

Fig.2

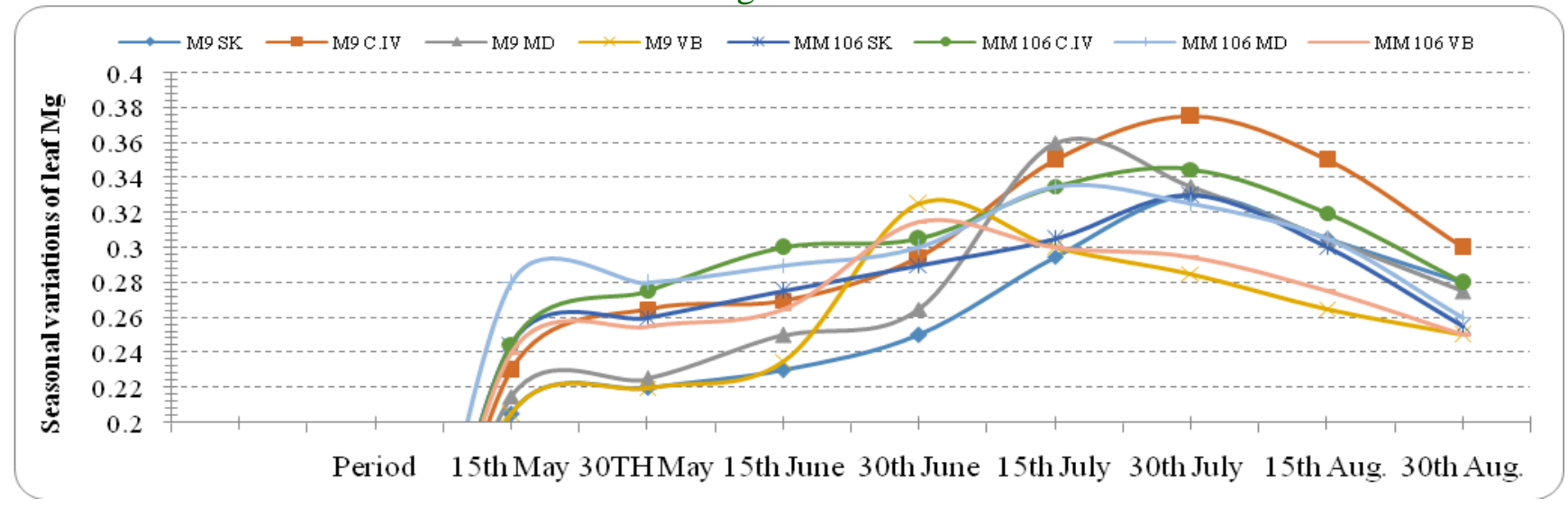

Fig.3

\section{Magnesium}

The comparison of leaf magnesium content between the two rootstocks depicted that significantly higher leaf magnesium content $(0.29$ and $0.29 \%)$ was recorded on $\mathrm{MM}_{106}$ rootstock compared to $\mathrm{M}_{9}(0.27$ and $0.28 \%)$ during the study period. Rootstock and variety effects on nutrient concentration of apple trees can be explained with the genetic effect leading to different nutrient uptake capacity (Kucukyumuk and Erdal, 2009). Among 
different cultivars significantly maximum leaf magnesium content $(0.29$ and $0.31 \%)$ was recorded in Cooper-IV, and minimum leaf magnesium content $(0.27 \%)$ was recorded in Vista Bella during both the years (table 03). Leaf magnesium content varied significantly among different sampling timings with maximum leaf magnesium content $(0.32 \%$ and $0.34 \%$ ) recorded on $30^{\text {th }}$ of July and minimum $(0.21 \%$ and $0.22 \%)$ was recorded on $15^{\text {th }}$ of May during both the years of experiment. There was an increasing trend in leaf magnesium content from $15^{\text {th }}$ of May to $30^{\text {th }}$ of July thereafter, decreased slowly upto $30^{\text {th }}$ of August. The nutrient content of leaves is subject to variation during the vegetation season and the level of calcium and magnesium in leaves of apple trees increases Tagliavini et al., (1992). The first phase occurs during the cellular division period, while the other phases are associated with the period of cellular expansion (Hilmelrick and McDuffie, 1983).

\section{Seasonal variation}

The obtained leaf magnesium content distribution, in practical terms, allowed to infer that leaf sampling for nutritional diagnosis might be anticipated for 30 days in relation to the period used in the present experiment and reported in the literature (14th to 18th week after full bloom), because there is a relative nutrient concentration stability in early maturing cultivar Vista Bella from $15^{\text {th }}$ of June to $15^{\text {th }}$ of July and Mollies Delicious showed the nutrient stability from $30^{\text {th }}$ of June to $30^{\text {th }}$ of July.

Whereas in mid-season maturing cultivars (Starkrimson and Cooper IV) from the $15^{\text {th }}$ July to $15^{\text {th }}$ of August. As the results indicated that the highest levels of magnesium concentrations were reached in July, midseason of vegetation, but through the end of the season, they began to decrease (Fig. 3).

\section{Fruit yield/plant (kg) and yield efficiency}

Fruit yield is prime concern to orchardists they grow the plants for better yield and good quality fruit production. The maximum fruit yield (16.55 kg/tree and $20.19 \mathrm{~kg} /$ tree) was recorded on $\mathrm{MM}_{106}$ rootstock were as the lowest $(13.79 \mathrm{~kg} /$ tree and $18.65 \mathrm{~kg} /$ tree) was records in $\mathrm{M}_{9}$ rootstock (table 04). Among the cultivars Vista Bella produced highest fruit yield (19.63 and $23.58 \mathrm{~kg} /$ tree) and Cooper IV produced the lowest $(7.28 \mathrm{~kg} /$ tree and 12.81 $\mathrm{kg} /$ tree). Among the genotypes $\mathrm{M}_{9}$ rootstock produced highest yield efficiency $(0.24$ $\mathrm{kg} / \mathrm{cm}^{-2}, 0.25 \mathrm{~kg} / \mathrm{cm}^{-2}$ ) as compared lowest value $\left(0.21 \mathrm{~kg} / \mathrm{cm}^{-2}, \quad 0.26 \mathrm{~kg} / \mathrm{cm}^{-2}\right)$ was recorded in $\mathrm{MM}_{106}$ rootstock. Vista Bella recorded the highest yield efficiency $(0.32$ $\mathrm{kg} / \mathrm{cm}^{-2}$ ) and Cooper IV recorded the lowest yield efficiency $\left(0.13 \mathrm{~kg} / \mathrm{cm}^{-2}\right)$ during both the years.

Fruit yield is of prime concern to orchardists they grow the plants for better yield and good quality fruit production. Yield is linearly related to light interception (Robinson, 2007). The linear relationship between yield and fruit count per tree and the sink strength of an apple crop is almost proportional to the number of fruit per tree (Hampson et al., 2002). Cultivars grafted on $\mathbf{M}_{9}$ had a more yield efficiency than $\mathrm{MM}_{106}$ increased photosynthesis and yield efficiency in grafted cultivars on dwarfing rootstock deriving from reduction of shoot growth Dorin et al., (2015).

\section{References}

Dorin, B., Mitre, I. J., Tripon, A., Mitre, I. and Mitre, V. 2015. The influence of cultivar, rootstock and culture system on growth and yield in apple. Bull. Horticult., 72(1): 33-38.

Erdal, I., Askin M. A., Kuçukyumuk, Z., Yildirim, F. and Yildirim, A. 2008. Rootstock has an important role on iron 
nutrition of apple trees. World J. Agricult. Sci., 4(2): 173-177.

Hampson, C. R., Quamm, H. A. and Brownlee, R. T. 2002. Canopy growth, yield and fruit quality of 'Royal Gala' apple trees grown for eight years in five tree training systems. J. Horticult. Sci., 37: 627-631.

Hirzel, J. F. and Best, S. 2009. Effect of two rootstock selections on the seasonal nutritional variability of Breaburn apple. The Proceedings of the International Plant Nutrition Colloquium. (Ed. U. C. Davis). XVI Poster No. 1375.

Hilmelrich, D. G. and McDuffie, R. F. 1983. The calcium cycle uptake and distribution in apple trees. Horticult. Sci., 18: 147151.

Jackson, M. L. 1973. Soil Chemical Analysis. $2^{\text {nd }}$ edition. Printice Hall of India, New Delhi pp. 498.

Jimenez, S., Pinochet, J., Gogorcena, Y., Betran, J. A. and Moreno, M. A. 2007. Influence of different vigour cherry rootstocks on leaves and shoots mineral composition. Scientia Horticulturae, 112: 73-79.

Jones, J. R., Wolf, J. B. and Milis, B. 1991. Plant Analysis Handbook. A Practical Sampling, Preparation, Analysis and Interpretion Guide. Micro-Macro Publishing, Inc. pp. 183.

Kamboj, J. S., Datt, A. S. and Rehalia, A. S. 1987. Standardization of leaf sampling technique in subtropical pear. Punjab Horticultural Journal 27(3-4): 121-132.

Kotur, S. C. and Singh, H. P. 1993. Leaf sampling techniques in litchi (litchi chinensis) Indian J. Agri. Sci., 63(10): 632-638.

Kucukyumuk, Z. and Erdal, I. 2009. Rootstock and variety effects on mineral nutrition of apple trees. Suleyman Demirel University, J. Faculty Agri., 4(2): 8-16.

Kucukyumuk, Z., Kucukyumuk, C., Erdal, I. and Eraslan, F. 2012. Seasonal variations and different irrigation programs on nutrient concentrations of 'Starkrimson Delicious' apple variety. World Acad. Sci. Engi. Technol., 71: 223-226.

Nachtigall, G. R. and Dechan, A. R. 2006. Seasonality of nutrients in leaves and fruits of apple trees. Science Agric. (Piracicaba, Braz.) 63(5): 493-501.

Rehalia, A. S. and Sandhu, R. D. 2005. Standardization of foliar sampling technique for macro-nutrient in persimmon Diasproskar L. cv. Hachiya. Acta Horticulturae, 696: 265-268.

Robinson, T. L. 2007. Recent advances and future directions in orchard planting systems. Acta Horticulturae, 732: 367382.

Roca-Perez, L., Boluda, R. and Bermudez, P. 2006. Seasonal Variation in Nutrient Status of Foxglove Leaves. J. Plant Nutr., 29(6): 1077-1084.

Schneider, G. W., Chaplin, C. E. and Martin, D. C. 1978. Effects of apple rootstock, tree spacing and cultivar on fruit and tree size, yield and foliar mineral composition. $J$. Am. Soc. Horticult. Sci., 103: 230-232.

Tagliavini, M., Scudellari, D., Marangoni, B., Bastianel, A., Franzin, F. and Zamborlini, M. 1992. Leaf mineral composition of apple tree sampling date and effects of cultivar and rootstock. J. Plant Nutr., 15(5): 605-619.

Wutscher, H. K. and Shull, A. V. 1976. Performance of Marrs Early orange on 11 rootstocks in South Texas. J. American Soc. Horticult. Sci., 101(2): 158-161.

\section{How to cite this article:}

Rather, J.A., F.A. Misgar, Z.A. Bhat, S. Sami Ullah, G.A. Dar and Javeed, K. 2018. Stionic Effect and Seasonal Nutrient Dynamics in Some Exotic Apple Cultivars. Int.J.Curr.Microbiol.App.Sci. 7(01): 10-19. doi: https://doi.org/10.20546/ijcmas.2018.701.002 